\title{
A Remark on Norm Inflation with General Initial Data for the Cubic Nonlinear Schrödinger Equations in Negative Sobolev Spaces
}

\author{
By \\ Tadahiro $\mathrm{OH}$ \\ (The University of Edinburgh, United Kingdom)
}

\begin{abstract}
In this note, we consider the ill-posedness issue for the cubic nonlinear Schrödinger equation. In particular, we prove norm inflation based at every initial condition in negative Sobolev spaces below or at the scaling critical regularity. inflation.

Key Words and Phrases. Nonlinear Schrödinger equation, Ill-posedness, Norm

2010 Mathematics Subject Classification Numbers. 35Q55.
\end{abstract}

\section{Introduction}

We consider the cubic nonlinear Schrödinger equation (NLS):

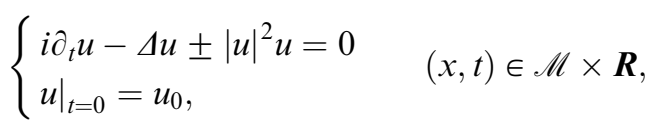

where $\mathscr{M}=\boldsymbol{R}^{d}$ or $\boldsymbol{T}^{d}$ with $\boldsymbol{T}=\boldsymbol{R} / \boldsymbol{Z}$. The equation (1.1) appears in various physical settings: nonlinear optics, fluids, plasmas, and quantum field theory, and has been studied extensively from both theoretical and applied points of view. See $[28,9,29]$ for a general review on the subject.

Our main goal in this paper is to study the ill-posedness issue of (1.1) in negative Sobolev spaces. We first recall the following scaling invariance for (1.1); if $u(x, t)$ is a solution to (1.1) on $\boldsymbol{R}^{d}$, then $u^{\lambda}(x, t):=\lambda^{-1} u\left(\lambda^{-1} x, \lambda^{-2} t\right)$ is also a solution to $(1.1)$ on $\boldsymbol{R}^{d}$ with scaled initial data. Associated to this scaling invariance, we have the critical Sobolev regularity given by $s_{\text {crit }}:=$ $d / 2-1$. While there is no scaling symmetry in the periodic setting, the heuristics provided by the scaling argument also plays an important role. It is commonly conjectured that an evolution equation is well-posed in $H^{s}$ for $s>s_{\text {crit }}$, while it is ill-posed for $s<s_{\text {crit }}$. In fact, we have a good well-posedness theory $[17,30,10,4,19,31]$ of $(1.1)$ for $s \geq s_{\text {crit }}$, at least locally in time, with the exception of the $d=1$ case. See Remark 1.5 below for a brief discussion on the situation when $d=1$. 
On the other hand, (1.1) is known to be ill-posed on both $\boldsymbol{R}^{d}$ and $\boldsymbol{T}^{d}$ when $s<s_{\text {crit }}$. When $d=1$, it is also ill-posed at the critical regularity $s=s_{\text {crit }}=$ $-1 / 2$. More precisely, we have the following norm inflation; given any $\varepsilon>0$, there exist a solution $u$ to $(1.1)$ on $\mathscr{M}$ and $t \in(0, \varepsilon)$ such that

$$
\|u(0)\|_{H^{s}(\mathscr{M})}<\varepsilon \quad \text { and } \quad\|u(t)\|_{H^{s}(\mathscr{M})}>\varepsilon^{-1} .
$$

See $[15,8,27,21]$. This is a stronger notion of ill-posedness than the failure of continuity of the solution map at 0 ; given any $\varepsilon>0$, there exist a solution $u$ to $(1.1)$ and $t \in(0, \varepsilon)$ such that

$$
\left\|u(0)-u_{0}\right\|_{H^{s}(\mathscr{M})}<\varepsilon \quad \text { and } \quad\|u(t)\|_{H^{s}(\mathscr{M})} \gtrsim 1,
$$

where $u_{0}=0$. Note that (the failure of) continuity at $u_{0}$ can be discussed for any $u_{0} \in H^{s}(\mathscr{M})$. In a similar manner, we can discuss norm inflation based at any $u_{0} \in H^{s}(\mathscr{M})$. This is precisely our goal in this paper.

Theorem 1.1. Given $d \in \boldsymbol{N}$, let $\mathscr{M}=\boldsymbol{R}^{d}$ or $\boldsymbol{T}^{d}$. Suppose that $s \in \boldsymbol{R}$ satisfies either (i) $s \leq-1 / 2$ when $d=1$ or (ii) $s<0$ when $d \geq 2$. Fix $u_{0} \in H^{s}(\mathscr{M})$. Then, given any $\varepsilon>0$, there exist a solution $u_{\varepsilon}$ to (1.1) on $\mathscr{M}$ and $t \in(0, \varepsilon)$ such that

$$
\left\|u_{\varepsilon}(0)-u_{0}\right\|_{H^{s}(\mathscr{M})}<\varepsilon \quad \text { and } \quad\left\|u_{\varepsilon}(t)\right\|_{H^{s}(\mathscr{M})}>\varepsilon^{-1} .
$$

When $u_{0}=0$, Theorem 1.1 is reduced to the usual norm inflation (based at the zero function) stated in (1.2). As a corollary to Theorem 1.1, we obtain the following discontinuity of the solution map.

Corollary 1.2. Let $(s, d)$ be as in Theorem 1.1. Then, for any $T>0$, the solution map $\Phi: u_{0} \in H^{s}(\mathscr{M}) \mapsto u \in C\left([-T, T] ; H^{s}(\mathscr{M})\right)$ to the cubic NLS (1.1) is discontinuous everywhere in $H^{s}(\mathscr{M})$.

Norm inflation based at general initial data was first studied in a recent paper by Xia [32] in the context of the nonlinear wave equations on $\boldsymbol{T}^{3}$. The argument in [32] is based on the (dispersionless) ODE approach in the spirit of Christ-Colliander-Tao [15] and Burq-Tzvetkov [6].

We prove Theorem 1.1 by a Fourier analytic approach. Recently, Iwabuchi-Ogawa [20] developed a method for proving ill-posedness of evolution equations, exploiting high-to-low energy transfer in the first Picard iterate. This method is built upon the previous work by Bejenaru-Tao [1] and is developed further to cope with a wider class of equations, utilizing modulation spaces. See Kishimoto [21] for a norm inflation result on (1.1) via this method. In the following, we implement a refinement of the argument in $[20,21]$ and prove Theorem 1.1. 
In $[20,21]$, the (scaled) modulation space $M_{2,1}$ and its algebra property played an important role. In the proof of Theorem 1.1, we simply use the Wiener algebra $\mathscr{F} L^{1}(\mathscr{M})$ as a replacement. Given $\mathscr{M}=\boldsymbol{R}^{d}$ or $\boldsymbol{T}^{d}$, let $\hat{\mathscr{M}}$ denote the Pontryagin dual of $\mathscr{M}$, i.e.

$$
\hat{\mathscr{M}}= \begin{cases}\boldsymbol{R}^{d} & \text { if } \mathscr{M}=\boldsymbol{R}^{d}, \\ \boldsymbol{Z}^{d} & \text { if } \mathscr{M}=\boldsymbol{T}^{d} .\end{cases}
$$

When $\hat{\mathscr{M}}=\boldsymbol{Z}^{d}$, we endow it with the counting measure. We then define the Fourier-Lebesgue space $\mathscr{F} L^{p}(\mathscr{M})$ by the norm:

$$
\|f\|_{\mathscr{F}^{p}(\mathscr{M})}=\|\hat{f}\|_{L^{p}(\mathscr{M})} .
$$

In particular, $\mathscr{F} L^{1}(\mathscr{M})$ corresponds to the Wiener algebra. By the algebra property, it is easy to see that (1.1) is analytically locally well-posed in $\mathscr{F} L^{1}(\mathscr{M})$.

Another key ingredient in $[20,21]$ is the power series expansion of a solution $u$ to $(1.1)$ with $\left.u\right|_{t=0}=u_{0}$ :

$$
u=\sum_{j=0}^{\infty} \Xi_{j}\left(u_{0}\right)
$$

where $\Xi_{j}\left(u_{0}\right)$ denotes homogeneous multilinear terms (in $u_{0}$ ) of degree $2 j+1$. In $[20,21], \Xi_{j}$ was defined by a recursive relation of the form:

$$
\Xi_{j}\left(u_{0}\right)=\sum_{\substack{j_{1}+j_{2}+j_{3}=j \\ j_{1}, j_{2}, j_{3} \geq 1}} \mathscr{I}\left[\Xi_{j_{1}}\left(u_{0}\right), \Xi_{j_{2}}\left(u_{0}\right), \Xi_{j_{3}}\left(u_{0}\right)\right],
$$

where $\mathscr{I}$ is the trilinear Duhamel integral operator defined in (2.1). Then, nonlinear estimates were proved inductively. In the following, we instead define $\Xi_{j}$ directly via the power series expansion indexed by trees. This allows us to establish nonlinear estimates without an induction. See Section 2. Such a power series expansion indexed by trees is more suitable in handling an expression such as $\Xi_{j}\left(u_{0}+\phi\right)-\Xi_{j}(\phi)$, which is the main new ingredient for showing norm inflation based at a general initial condition $u_{0}$.

When $d \geq 3$, we have $s_{\text {crit }}>0$ and thus Theorem 1.1 leaves a gap $\left(0, s_{\text {crit }}\right)$. In view of the mass conservation, (1.3) can not hold for $s=0$. Moreover, when $s_{\text {crit }}>0$, we do not have norm inflation at $s=s_{\text {crit }}$ in view of the wellposedness in the scaling critical spaces. In this case, one needs to exploit lowto-high energy transfer to prove an analogue of Theorem 1.1 for $s \in\left(0, s_{\text {crit }}\right)$. By employing the ODE approach as in $[15,27]$, we plan to address this issue in a forthcoming work.

We conclude this introduction by several remarks. 
Remark 1.3. We point out that Theorem 1.1 also holds on a general irrational torus $\boldsymbol{T}_{\alpha}^{d}=\prod_{j=1}^{d}\left(\boldsymbol{R} / \alpha_{j} \boldsymbol{Z}\right), \alpha_{j}>0$. This is due to the fact that the proof of Theorem 1.1 does not use any fine arithmetic property of frequency interactions. Indeed, we only use the following trivial estimate:

$$
\left.|| \xi\right|^{2}-\left|\xi_{1}\right|^{2}+\left|\xi_{2}\right|^{2}-\left|\xi_{3}\right|^{2} \mid \lesssim N^{2}
$$

for $\xi=\xi_{1}-\xi_{2}+\xi_{3}$ with $\left|\xi_{j}\right| \lesssim N, j=1,2,3$. See (3.18) below.

Remark 1.4. In Theorem 1.1, we only considered the cubic nonlinearity for simplicity of the presentation. Our method is elementary and can be applied to other power-type nonlinearities.

We can also consider norm inflation based at general initial data for the cubic fractional NLS:

$$
i \partial_{t} u+\left(-\Delta^{2}\right)^{\alpha} u \pm|u|^{2} u=0 .
$$

In [11], Choffrut-Pocovnicu recently applied the argument in $[20,21]$ and proved norm inflation (1.2) (at the zero function) for the cubic half-wave equation (i.e. $\alpha=1 / 2$ ) on $\boldsymbol{R}$ and $\boldsymbol{T}$ when $s<0$. By adapting the proof of Theorem 1.1, we can extend this result in [11] to norm inflation based at general initial data as in Theorem 1.1.

After the completion of this manuscript, the author has learned that Choffrut and Pocovnicu [11] extended their norm inflation result to the general cubic fractional NLS for any $\alpha>0$. In particular when $\alpha>1$, they established norm inflation above the scaling critical regularity.

Remark 1.5. The equation (1.1) is also invariant under the Galilean symmetry. This symmetry preserves the $L^{2}$-norm of solutions, giving rise to another critical regularity $s_{\text {crit }}^{\infty}:=0$. In particular, this critical regularity plays an important role when $d=1$. While (1.1) is globally well-posed in $L^{2}(\boldsymbol{R})$ [30], it is known to be 'mildly ill-posed' below $L^{2}(\boldsymbol{R})$ in the sense of the failure of local uniform continuity in negative Sobolev spaces $[22,13]$.

On $\boldsymbol{T}$, the contrast between $s \geq 0$ and $s<0$ is more drastic. On the one hand, (1.1) is globally well-posed in $L^{2}(\boldsymbol{T})$ [4]. On the other hand, it is illposed in negative Sobolev spaces. Christ-Colliander-Tao [14] and Molinet [25] showed discontinuity of the solution map in negative Sobolev spaces. Moreover, Guo-Oh [18] proved non-existence of solutions for (1.1) with initial data lying strictly in a negative Sobolev space.

As an alternative model to (1.1) in low regularity setting on $\boldsymbol{T}^{d}$, we have the following Wick ordered cubic NLS:

$$
i \partial_{t} u-\Delta u \pm\left(|u|^{2}-2 \int_{T^{d}}|u|^{2} d x\right) u=0 .
$$


This equation is gauge equivalent to $(1.1)$ in $L^{2}\left(\boldsymbol{T}^{d}\right)$, while it behaves better than (1.1) outside $L^{2}\left(\boldsymbol{T}^{d}\right)$. See [26] for more discussion on this issue. By slightly modifying the argument, Theorem 1.1 also holds for (1.6) on $\boldsymbol{T}^{d}$. See Remark 3.6.

Lastly, we point out that the well-posedness issue of (1.1) on $\boldsymbol{R}$ and of (1.6) on $\boldsymbol{T}$ in $H^{s}$ for $-1 / 2<s<0$ is widely open. See $[23,16,24,18]$ for partial results on this problem.

Remark 1.6. Given initial data below a scaling critical regularity $s_{\text {crit }}$, it is often possible to suitably randomize initial data and construct solutions in a probabilistic manner. See $[5,6,2,3]$. Such probabilistic well-posedness results do not yield (deterministic) continuous dependence in $H^{s}$ with $s<s_{\text {crit }}$. See also [7] for the notion of probabilistic continuous dependence. In particular, norm inflation based at general data such as Theorem 1.1 does not contradict these probabilistic results.

It is worthwhile to note that these probabilistic constructions of solutions yield mild continuous dependence in a smoother regularity. Given $u_{0} \in H^{s}$ with $s<s_{\text {crit }}$, the argument in $[5,6,2,3]$ allows us to randomize $u_{0}$ and construct a set $\Sigma_{u_{0}}$ of full probability such that, given any $\omega \in \Sigma_{u_{0}}$, there exists a random solution $u^{\omega}$ of the form $u^{\omega}=S(t) u_{0}^{\omega}+v^{\omega}$, where $S(t) u_{0}^{\omega}$ is the linear solution with the randomized initial data $u_{0}^{\omega}$ and $v^{\omega}$ denotes the random nonlinear part belonging to a smoother space; $v^{\omega}(t) \in H^{\sigma}$ for some $\sigma \geq s_{\text {crit }}$. Moreover, there exists $\varepsilon_{0}>0$ such that if a deterministic function $w_{0}$ satisfies

$$
\left\|u_{0}^{\omega}-w_{0}\right\|_{H^{\sigma}}<\varepsilon_{0}
$$

for some $\omega \in \Sigma_{u_{0}}$, then there exists a solution $w$ with $\left.w\right|_{t=0}=w_{0}$, satisfying

$$
\left\|u^{\omega}(t)-w(t)\right\|_{H^{\sigma}} \lesssim\left\|u_{0}^{\omega}-w_{0}\right\|_{H^{\sigma}}
$$

for $t \in\left[-T_{\omega}, T_{\omega}\right]$. Namely, we have some kind of continuous dependence in this probabilistic setting, but in a smoother regularity. Note that this mild continuous dependence (1.8) does not contradict Theorem 1.1 since they are in different regularity regimes: $s<s_{\text {crit }} \leq \sigma$. In particular, (1.7) is much more restrictive than the first estimate in (1.3).

The defocusing/focusing nature of the equation does not play any role. Hence, we assume that it is defocusing (with the +sign in (1.1)) in the following. Moreover, in view of the time reversibility of the equation, we only consider positive times. 


\section{Preliminary analysis}

In this section, we first discuss the local well-posedness of (1.1) in the Wiener algebra $\mathscr{F} L^{1}$. Then, we express solutions in power series in terms of initial data, where the summation ranges over all finite ternary trees. We then establish basic nonlinear estimates on the multilinear terms arising in the power series expansion.

\subsection{Power series expansion indexed by trees}

We define the Duhamel integral operator $\mathscr{I}$ by

$$
\mathscr{I}\left[u_{1}, u_{2}, u_{3}\right](t):=i \int_{0}^{t} S\left(t-t^{\prime}\right)\left[u_{1}\left(t^{\prime}\right) \overline{u_{2}\left(t^{\prime}\right)} u_{3}\left(t^{\prime}\right)\right] d t^{\prime},
$$

where $S(t)=e^{-i t \Delta}$ is the linear Schrödinger propagator. When all the three arguments $u_{1}, u_{2}$, and $u_{3}$ are identical, we use the following shorthand notation:

$$
\mathscr{I}^{3}[u]:=\mathscr{I}[u, u, u] .
$$

We say that $u$ is a solution to (1.1) with $\left.u\right|_{t=0}=u_{0}$ if $u$ satisfies the following Duhamel formulation:

$$
u(t)=S(t) u_{0}+\mathscr{I}^{3}[u](t) .
$$

We first state the local well-posedness of $(1.1)$ in the Wiener algebra $\mathscr{F} L^{1}$.

Lemma 2.1. The cubic NLS (1.1) is locally well-posed in the Wiener algebra $\mathscr{F} L^{1} . \quad$ More precisely, given $u_{0} \in \mathscr{F} L^{1}$, there exist $T \sim\left\|u_{0}\right\|_{\mathscr{F} L^{1}}^{-2}>0$ and a unique solution $u \in C\left([-T, T] ; \mathscr{F} L^{1}\right)$ satisfying (2.3).

In view of the unitarity of $S(t)$ in $\mathscr{F} L^{1}$ and the algebra property of $\mathscr{F} L^{1}$, Lemma 2.1 follows from a standard fixed point argument. We omit details.

Let $\phi \in \mathscr{F} L^{1}$. Then, (the proof of) Lemma 2.1 guarantees the convergence of the following Picard iteration scheme:

$$
P_{0}(\phi)=S(t) \phi \quad \text { and } \quad P_{j}(\phi)=S(t) \phi+\mathscr{I}^{3}\left[P_{j-1}(\phi)\right], \quad j \in N,
$$

at least for short times. It follows from (2.2) and (2.4) that $P_{j}$ consists of multilinear terms of degrees at most $3^{j}$ (in $\left.\phi\right)$. In the following, we discuss a more general recursive scheme and express a solution in a power series indexed by trees. Christ [12] implemented such a power series expansion of solutions to the Wick ordered cubic NLS (1.6) on $\boldsymbol{T}$ in a low regularity setting. Since we work only with smooth functions, our presentation is much simpler.

We introduce the following notion of (ternary) trees. As in [12], our trees refer to a particular subclass of usual trees with the following properties: 
Definition 2.2. (i) Given a partially ordered set $\mathscr{T}$ with partial order $\leq$, we say that $b \in \mathscr{T}$ with $b \leq a$ and $b \neq a$ is a child of $a \in \mathscr{T}$, if $b \leq c \leq a$ implies either $c=a$ or $c=b$. If the latter condition holds, we also say that $a$ is the parent of $b$.

(ii) A tree $\mathscr{T}$ is a finite partially ordered set, satisfying the following properties:

- Let $a_{1}, a_{2}, a_{3}, a_{4} \in \mathscr{T}$. If $a_{4} \leq a_{2} \leq a_{1}$ and $a_{4} \leq a_{3} \leq a_{1}$, then we have $a_{2} \leq a_{3}$ or $a_{3} \leq a_{2}$

- A node $a \in \mathscr{T}$ is called terminal, if it has no child. A non-terminal node $a \in \mathscr{T}$ is a node with exactly three children,

- There exists a maximal element $r \in \mathscr{T}$ (called the root node) such that $a \leq r$ for all $a \in \mathscr{T}$,

- $\mathscr{T}$ consists of the disjoint union of $\mathscr{T}^{0}$ and $\mathscr{T}^{\infty}$, where $\mathscr{T}^{0}$ and $\mathscr{T}^{\infty}$ denote the collections of non-terminal nodes and terminal nodes, respectively.

Note that the number $|\mathscr{T}|$ of nodes in a tree $\mathscr{T}$ is $3 j+1$ for some $j \in N \cup\{0\}$, where $\left|\mathscr{T}^{0}\right|=j$ and $\left|\mathscr{T}^{\infty}\right|=2 j+1$. Let us denote the collection of trees in the $j$ th generation (i.e. with $j$ parental nodes) by $\mathbf{T}(j)$, i.e.

$$
\mathbf{T}(j):=\{\mathscr{T}: \mathscr{T} \text { is a tree with }|\mathscr{T}|=3 j+1\} .
$$

Then, we have the following exponential bound on the number \# $\mathbf{T}(j)$ of trees in the $j$ th generation.

Lemma 2.3. Let $\mathbf{T}(j)$ be as above. Then, there exists $C_{0}>0$ such that

$$
\# \mathbf{T}(j) \leq C_{0}^{j}
$$

for all $j \in N \cup\{0\}$.

This lemma is a basic fact about ternary trees. We present the proof for the sake of completeness.

Proof. Clearly, we have $\# \mathbf{T}(0)=\# \mathbf{T}(1)=1$. We now consider $j \geq 2$. From Definition 2.2, we have the following identity:

$$
\# \mathbf{T}(j)=\sum_{\substack{j_{1}+j_{2}+j_{3}=j-1 \\ j_{1}, j_{2}, j_{3} \geq 0}} \# \mathbf{T}\left(j_{1}\right) \cdot \# \mathbf{T}\left(j_{2}\right) \cdot \# \mathbf{T}\left(j_{3}\right)
$$

Assume the following stronger estimate:

$$
\# \mathbf{T}(k) \leq \frac{C_{0}^{k}}{(1+k)^{2}}
$$


for all $k \leq j-1$. Then, from (2.6) and (2.7) with $3 \max \left(j_{1}+1, j_{2}+1, j_{3}+1\right)$ $\geq j+1$, we have

$$
\begin{aligned}
\# \mathbf{T}(j) & \leq \sum_{\substack{j_{1}+j_{2}+j_{3}=j-1 \\
j_{1}, j_{2}, j_{3} \geq 0}} \frac{C_{0}^{j_{1}}}{\left(1+j_{1}\right)^{2}} \frac{C_{0}^{j_{2}}}{\left(1+j_{2}\right)^{2}} \frac{C_{0}^{j_{3}}}{\left(1+j_{3}\right)^{2}} \\
& \leq 3^{2}\left(\sum_{k \geq 0} \frac{1}{(1+k)^{2}}\right)^{2} \cdot \frac{C_{0}^{j-1}}{(1+j)^{2}} \leq \frac{C_{0}^{j}}{(1+j)^{2}},
\end{aligned}
$$

where the last inequality holds by choosing $C_{0}=3^{2}\left(\sum_{k \geq 0} 1 /(1+k)^{2}\right)^{2}<\infty$. This proves (2.7) for $k=j$. By induction, (2.7) holds for all $j \in \boldsymbol{N} \cup\{0\}$, yielding (2.5) for all $j \in N \cup\{0\}$.

Remark 2.4. In [21], a similar counting argument is needed to control the number of terms appearing in the recursive definition (1.5) of $\Xi_{j}$.

Next, we express the solution $u$ constructed in Lemma 2.1 in a power series indexed by trees. Fix $\phi \in \mathscr{F} L^{1}$. Given a tree $\mathscr{T} \in \mathbf{T}(j), j \in \boldsymbol{N} \cup\{0\}$, we associate a multilinear operator (in $\phi$ ) by the following rules:

- Replace a non-terminal node "o" by the Duhamel integral operator $\mathscr{I}$ defined in $(2.1)$ with its three children as arguments $u_{1}, u_{2}$, and $u_{3}$

- Replace a terminal node "." by the linear solution $S(t) \phi$.

By a multilinear operator, we mean an operator which is linear or conjugate linear with respect to each argument, i.e. linear over real numbers. In the following, we denote this mapping from $\bigcup_{j=0}^{\infty} \mathbf{T}(j)$ to $\mathscr{D}^{\prime}(\mathscr{M} \times[-T, T])$ by $\Psi_{\phi}$.

For example, $\Psi_{\phi}$ maps the trivial tree ".", consisting only of the root node to the linear solution $S(t) \phi$. Namely, we have $\Psi_{\phi}(\cdot)=S(t) \phi$. Similarly, we have

$$
\begin{aligned}
\Psi_{\phi}(\AA) & =\mathscr{I}^{3}[S(t) \phi], \\
\Psi_{\phi}\left(\AA^{\Re}\right) & =\mathscr{I}\left[\mathscr{I}^{3}[S(t) \phi], S(t) \phi, S(t) \phi\right],
\end{aligned}
$$

where $\mathscr{I}^{3}$ is as in (2.2). In view of the algebra property of $\mathscr{F} L^{1}$ along with the continuity and unitarity of $S(t)$, we have $\Psi_{\phi}(\mathscr{T}) \in C\left([-T, T] ; \mathscr{F} L^{1}\right)$ for any tree $\mathscr{T}$, provided $\phi \in \mathscr{F} L^{1}$. Note that, if $\mathscr{T} \in \mathbf{T}(j)$, then $\Psi_{\phi}(\mathscr{T})$ is $(2 j+1)$-linear in $\phi$. Lastly, we define $\Xi_{j}$ by

$$
\Xi_{j}(\phi):=\sum_{\mathscr{T} \in \mathbf{T}(j)} \Psi_{\phi}(\mathscr{T})
$$


Now, let us extend the definition of $\Psi_{\phi}$ by adding the following rules for another kind of terminal node "»":

- Replace a star-shaped terminal node " " by the solution $u$,

- Extend the definition of $\Psi_{\phi}$ to formal sums of trees by imposing linearity.

Then, the Duhamel formulation (2.3) can be represented by

$$
\text { - }=+ \text {. A. }
$$

By recursively applying (2.11) and eliminating the occurrence of " " from younger trees, we have

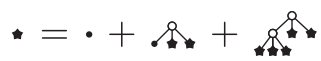

$$
\begin{aligned}
& =\cdot+\AA+\infty+\infty \\
& =\cdot+\AA+\Omega+R+\Omega
\end{aligned}
$$

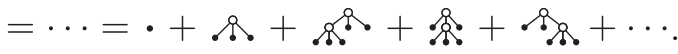

This iterative scheme leads, under $\Psi_{\phi}$, to the following (formal) power series expansion of the solution $u$ to (1.1) with $\left.u\right|_{t=0}=\phi \in \mathscr{F} L^{1}$ :

$$
\begin{aligned}
u & =\sum_{j=0}^{\infty} \Xi_{j}(\phi)=\sum_{j=0}^{\infty} \sum_{\mathscr{T} \in \mathbf{T}(j)} \Psi_{\phi}(\mathscr{T}) \\
& =\Psi_{\phi}(\cdot)+\Psi_{\phi}(\AA)+\Psi_{\phi}(\AA)+\Psi_{\phi}(\stackrel{\AA}{\AA})+\Psi_{\phi}(\stackrel{\AA}{\AA})+\cdots .
\end{aligned}
$$

It is easy to see that the above power series converges (absolutely) in $C\left([-T, T] ; \mathscr{F} L^{1}\right)$ and the first equality holds as long as $T=T\left(\|\phi\|_{\mathscr{F} L^{1}}\right) \sim$ $\|\phi\|_{\mathscr{F} L^{1}}^{-2}>0$ is sufficiently small. This follows from Lemma 2.5 below along with Lemma 2.3. See also [12, Proposition 6.4.2].

Given a tree $\mathscr{T} \in \mathbf{T}(j)$, label its terminal nodes by $a_{1}, \ldots, a_{2 j+1}$ (say, by moving from left to right in the planar graphical representation of the tree). Given functions $\phi_{1}, \ldots, \phi_{2 j+1} \in \mathscr{F} L^{1}$, we also define $\Psi\left(\mathscr{T} ; \phi_{1}, \ldots, \phi_{2 j+1}\right)$ by the following rules:

- Replace a non-terminal node $a \in \mathscr{T}^{0}$ by the Duhamel integral operator $\mathscr{I}$ with its three children as arguments $u_{1}, u_{2}$, and $u_{3}$,

- Replace terminal nodes $a_{k} \in \mathscr{T}^{\infty}$ by the linear solutions $S(t) \phi_{k}, k=$ $1, \ldots, 2 j+1$.

In particular, we have $\Psi_{\phi}(\mathscr{T})=\Psi(\mathscr{T} ; \phi, \ldots, \phi)$.

\subsection{Basic multilinear estimates}

In this subsection, we state basic multilinear estimates on $\Xi_{j}$. These will be used in establishing $H^{s}$-estimates on $\Xi_{j}\left(u_{0}+\phi\right)$ in Section 3 . 
Lemma 2.5. There exists $C>0$ such that

$$
\begin{aligned}
& \left\|\Xi_{j}(\phi)(t)\right\|_{\mathscr{F} L^{1}} \leq C^{j} t^{j}\|\phi\|_{\mathscr{F} L^{1}}^{2 j+1}, \\
& \left\|\Xi_{j}(\phi)(t)\right\|_{\mathscr{F} L^{\infty}} \leq C^{j} t^{j}\|\phi\|_{\mathscr{F} L^{1}}^{2 j-1}\|\phi\|_{L^{2}}^{2}
\end{aligned}
$$

for all $\phi \in \mathscr{F} L^{1}(\mathscr{M})$ and all $j \in N$.

Proof. Given $\mathscr{T} \in \mathbf{T}(j), \Psi_{\phi}(\mathscr{T})$ contains $j=\left|\mathscr{T}^{0}\right|$ (partially) iterated time integrations over a subset of $[0, t]^{j}$. Then, from the unitarity of $S(t)$ in $\mathscr{F} L^{1}$ and $L^{2}$ and Young's inequality, we have

$$
\begin{aligned}
& \left\|\Psi_{\phi}(\mathscr{T})(t)\right\|_{\mathscr{F} L^{1}} \lesssim t^{j}\|\phi\|_{\mathscr{\mathscr { F } L} L^{1}}^{2 j+1}, \\
& \left\|\Psi_{\phi}(\mathscr{T})(t)\right\|_{\mathscr{F} L^{\infty}} \lesssim t^{j}\|\phi\|_{\mathscr{F} L^{1}}^{2 j-1}\|\phi\|_{L^{2}}^{2}
\end{aligned}
$$

Hence, the desired estimates (2.13) and (2.14) follow from (2.15) and (2.16) with (2.10) and Lemma 2.3.

Lemma 2.6. There exists $C>0$ such that

$$
\left\|\Xi_{j}\left(u_{0}+\phi\right)(t)-\Xi_{j}(\phi)(t)\right\|_{\mathscr{F} L^{p}} \leq C^{j} t^{j}\left\|u_{0}\right\|_{\mathscr{F} L^{p}}\left(\left\|u_{0}\right\|_{\mathscr{F} L^{1}}^{2 j}+\|\phi\|_{\mathscr{F} L^{1}}^{2 j}\right)
$$

for all $u_{0} \in \mathscr{F} L^{p}(\mathscr{M}) \cap \mathscr{F} L^{1}(\mathscr{M})$ with $1 \leq p \leq \infty, \phi \in \mathscr{F} L^{1}(\mathscr{M})$, and $j \in N$.

Proof. From (2.10) and the multilinearity of $\Psi_{\phi}(\mathscr{T})$ in $\phi$, we have

$$
\begin{aligned}
\Xi_{j}\left(t ; u_{0}+\phi\right)-\Xi_{j}(\phi) & =\sum_{\mathscr{T} \in \mathbf{T}(j)}\left(\Psi_{u_{0}+\phi}(\mathscr{T})-\Psi_{\phi}(\mathscr{T})\right) \\
& =\sum_{\mathscr{T} \in \mathbf{T}(j)} \sum_{\phi_{1}, \ldots, \phi_{2 j+1}} \Psi\left(\mathscr{T} ; \phi_{1}, \ldots, \phi_{2 j+1}\right) .
\end{aligned}
$$

Here, the second summation in $\phi_{1}, \ldots, \phi_{2 j+1}$ takes over all possible combinations of $\phi_{k}=u_{0}$ or $\phi_{k}=\phi$ with at least one occurrence of $u_{0}$. Note that we have

$$
\left|\sum_{\phi_{1}, \ldots, \phi_{2 j+1}} 1\right| \leq 2^{2 j+1}
$$

As in the proof of Lemma 2.5, given $\mathscr{T} \in \mathbf{T}(j)$, there are $j=\left|\mathscr{T}^{0}\right|$ time integrations in $\Psi\left(\mathscr{T} ; \phi_{1}, \ldots, \phi_{2 j+1}\right)$ over a subset of $[0, t]^{j}$. Then, from the unitarity of $S(t)$ in $\mathscr{F} L^{p}$ and $\mathscr{F} L^{1}$ and Young's inequality, we have

$$
\left\|\Psi\left(\mathscr{T} ; \phi_{1}, \ldots, \phi_{2 j+1}\right)(t)\right\|_{\mathscr{F} L^{p}} \lesssim t^{j}\left\|\phi_{k_{*}}\right\|_{\mathscr{F} L^{p}} \prod_{\substack{k=1 \\ k \neq k_{*}}}^{2 j+1}\left\|\phi_{k}\right\|_{\mathscr{F} L^{1}} .
$$


Then, the desired estimate (2.17) follows from (2.18), (2.19), (2.20), and Lemma 2.3 .

\section{Proof of Theorem 1.1}

In this section, we present the proof of Theorem 1.1. We first reduce Theorem 1.1 to the following proposition. Given $\mathscr{M}=\boldsymbol{R}^{d}$ or $\boldsymbol{T}^{d}$, we use $\mathscr{S}(\mathscr{M})$ to denote the class of Schwartz functions if $\mathscr{M}=\boldsymbol{R}^{d}$ and the class of $C^{\infty}$-functions if $\mathscr{M}=\boldsymbol{T}^{d}$.

Proposition 3.1. Let $(s, d)$ be as in Theorem 1.1. Fix $u_{0} \in \mathscr{S}(\mathscr{M})$. Then, given any $n \in N$, there exist a solution $u_{n}$ to $(1.1)$ and $t_{n} \in(0,1 / n)$ such that

$$
\left\|u_{n}(0)-u_{0}\right\|_{H^{s}(\mathscr{M})}<\frac{1}{n} \quad \text { and } \quad\left\|u_{n}\left(t_{n}\right)\right\|_{H^{s}(\mathscr{M})}>n .
$$

We first prove Theorem 1.1, assuming Proposition 3.1. Given $u_{0} \in H^{s}$, let $\left\{u_{0, k}\right\}_{k \in N} \subset \mathscr{S}(\mathscr{M})$ be a sequence of smooth functions converging to $u_{0}$ in $H^{s}$, satisfying

$$
\left\|u_{0, k}-u_{0}\right\|_{H^{s}(\mathscr{M})}<\frac{1}{k}
$$

It follows from Proposition 3.1 that, for each $k \in N$, there exists a sequence $\left\{u_{k, n}\right\}_{n \in N}$ of solutions to (1.1) such that

$$
\left\|u_{k, n}(0)-u_{0, k}\right\|_{H^{s}(\mathscr{M})}<\frac{1}{n} \quad \text { and } \quad\left\|u_{k, n}\left(t_{n}\right)\right\|_{H^{s}(\mathscr{M})}>n
$$

for all $n \in N$. Now, given $\varepsilon>0$, set $u_{\varepsilon}=u_{n, n}$ for some $n \geq 2 \varepsilon^{-1}$. Then, (1.3) follows from (3.2) and (3.3). This proves Theorem 1.1.

The remaining part of this paper is devoted to the proof of Proposition 3.1. In the following, we fix $u_{0} \in \mathscr{S}(\mathscr{M})$ and may suppress the dependence of various constants on $u_{0}$.

\subsection{Multilinear estimates}

In this subsection, we establish elementary, yet important multilinear estimates in the relevant Sobolev $H^{s}$-norm. In particular, Proposition 3.4 provides a lower bound on the trilinear term $\Xi_{1}\left(\phi_{n}\right)$ for appropriately chosen functions $\phi_{n}$.

Given $n \in N$, fix $N=N(n) \gg 1$ (to be chosen later). We define $\phi_{n}$ by setting

$$
\hat{\phi}_{n}(\xi)=R\left\{\mathbf{1}_{N e_{1}+Q_{A}}(\xi)+\mathbf{1}_{2 N e_{1}+Q_{A}}(\xi)\right\},
$$


where $Q_{A}=[-A / 2, A / 2)^{d}, e_{1}=(1,0, \ldots, 0), R=R(N) \geq 1$, and $A=A(N) \gg 1$, satisfying

$$
R A^{d} \gg\left\|u_{0}\right\|_{\mathscr{F} L^{1}}, \quad \text { and } \quad A \ll N,
$$

are to be chosen later. Note that we have

$$
\left\|\phi_{n}\right\|_{H^{s}} \sim R A^{d / 2} N^{s} \quad \text { and } \quad\left\|\phi_{n}\right\|_{\mathscr{F} L^{1}} \sim R A^{d},
$$

for any $s \in \boldsymbol{R}$. Lastly, set

$$
u_{0, n}=u_{0}+\phi_{n} .
$$

Let $u_{n}$ be the corresponding solution to (1.1) with $\left.u_{n}\right|_{t=0}=u_{0, n}$. Lemmas 2.3 and 2.5 with (3.6) guarantee the convergence of the following power series expansion:

$$
u_{n}=\sum_{j=0}^{\infty} \Xi_{j}\left(u_{0, n}\right)=\sum_{j=0}^{\infty} \Xi_{j}\left(u_{0}+\phi_{n}\right),
$$

on $[-T, T]$, as long as

$$
T \lesssim\left(\left\|u_{0}\right\|_{\mathscr{F} L^{1}}+R A^{d}\right)^{-2} \sim\left(R A^{d}\right)^{-2},
$$

where the last equivalence follows from (3.5). Our main goal is to show that $u_{n}$ satisfies $(3.1)$ by estimating each of $\Xi_{j}\left(u_{0, n}\right)$ in (3.8).

We now state the nonlinear estimates. Keep in mind that implicit constants in Lemmas 3.2 and 3.3 depend on (various norms of) $u_{0}$.

Lemma 3.2. Let $\phi_{n}$ and $u_{0, n}$ be as in (3.4) and (3.7). Let $s<0$. Then, the following estimates hold:

$$
\begin{gathered}
\left\|u_{0, n}-u_{0}\right\|_{H^{s}} \lesssim R A^{d / 2} N^{s}, \\
\left\|\Xi_{0}\left(u_{0, n}\right)\right\|_{H^{s}} \lesssim 1+R A^{d / 2} N^{s} \\
\left\|\Xi_{1}\left(u_{0, n}\right)(t)-\Xi_{1}\left(\phi_{n}\right)(t)\right\|_{H^{s}} \lesssim t\left\|u_{0}\right\|_{L^{2}} R^{2} A^{2 d} .
\end{gathered}
$$

Proof. Noting that $\phi_{n}=u_{0, n}-u_{0}$, the first two estimates (3.9) and (3.10) follow from (3.6) and the unitarity of $S(t)$. Since $s<0$, it follows from Lemma 2.6 (with $p=2$ ) with (3.6) and (3.5) that

$$
\begin{aligned}
\left\|\Xi_{1}\left(u_{0, n}\right)(t)-\Xi_{1}\left(\phi_{n}\right)(t)\right\|_{H^{s}} & \lesssim t\left\|u_{0}\right\|_{L^{2}}\left(\left\|u_{0}\right\|_{\mathscr{F} L^{1}}^{2}+\left\|\phi_{n}\right\|_{\mathscr{F} L^{1}}^{2}\right) \\
& \lesssim t\left\|u_{0}\right\|_{L^{2}}\left(1+R^{2} A^{2 d}\right) \\
& \lesssim t\left\|u_{0}\right\|_{L^{2}} R^{2} A^{2 d}
\end{aligned}
$$

This proves (3.11). 
Lemma 3.3. Let $\phi_{n}$ and $u_{0, n}$ be as in (3.4) and (3.7). Let $s<0$. Then, there exists $C>0$ such that

$$
\left\|\Xi_{j}\left(u_{0, n}\right)(t)\right\|_{H^{s}} \leq C^{j} t^{j}\left(R A^{d}\right)^{2 j}\left\{R f(A)+\left\|u_{0}\right\|_{L^{2}}\right\}
$$

for any $j \in N$, where $f(A)$ is given by

$$
f(A)= \begin{cases}1, & \text { if } s<-\frac{d}{2}, \\ (\log A)^{1 / 2}, & \text { if } s=-\frac{d}{2}, \\ A^{d / 2+s} & \text { if } s>-\frac{d}{2} .\end{cases}
$$

Proof. From (3.4), we see that supp $\hat{\phi}_{n}$ consists of two disjoint cubes of volume $\sim A^{d}$. Given $\mathscr{T} \in \mathbf{T}(j), \Psi_{\phi}(\mathscr{T})$ is basically a $(2 j+1)$-fold product of $S(t) \phi$ and its complex conjugate under some integral operator in time. Hence, the spatial support of $\mathscr{F}\left[\Psi_{\phi}(\mathscr{T})\right]$ consists of (at most) $2^{2 j+1}$ cubes of volume $\sim A^{d}$. Then, from (2.10) and Lemma 2.3, we have

$$
\left|\operatorname{supp} \mathscr{F}\left[\Xi_{j}\left(\phi_{n}\right)\right]\right| \leq C^{j} A^{d} \leq c\left|C^{j} Q_{A}\right|
$$

for some $c, C>0$. Since $s<0,\langle\xi\rangle^{s}$ is a decreasing function in $|\xi|$. Hence, we obtain

$$
\begin{aligned}
\left\|\langle\xi\rangle^{s}\right\|_{L_{\xi}^{2}\left(\operatorname{supp} \mathscr{F}\left[\Xi_{j}\left(\phi_{n}\right)\right]\right)} & \leq\left\|\langle\xi\rangle^{s}\right\|_{L_{\xi}^{2}\left(c C^{j} Q_{A}\right)} \\
& \lesssim \begin{cases}1, & \text { if } s<-\frac{d}{2}, \\
C^{j}(\log A)^{1 / 2}, & \text { if } s=-\frac{d}{2}, \\
C^{j} A^{d / 2+s} & \text { if } s>-\frac{d}{2} .\end{cases}
\end{aligned}
$$

By Lemma 2.5 with (3.6) and (3.14), we have

$$
\begin{aligned}
\left\|\Xi_{j}\left(\phi_{n}\right)(t)\right\|_{H^{s}} & \leq\left\|\langle\xi\rangle^{s}\right\|_{L_{\xi}^{2}\left(\operatorname{supp} \mathscr{F}\left[\Xi_{j}\left(\phi_{n}\right)\right]\right)}\left\|\Xi_{j}\left(\phi_{n}\right)(t)\right\|_{\mathscr{F} L^{\infty}} \\
& \leq C^{j} t^{j}\left(R A^{d}\right)^{2 j} R f(A) .
\end{aligned}
$$

On the other hand, by Lemma 2.6 with (3.6) and (3.5), we have

$$
\begin{aligned}
\left\|\Xi_{j}\left(u_{0}+\phi_{n}\right)(t)-\Xi_{j}\left(\phi_{n}\right)(t)\right\|_{H^{s}} & \leq\left\|\Xi_{j}\left(u_{0}+\phi_{n}\right)(t)-\Xi_{j}\left(\phi_{n}\right)(t)\right\|_{L^{2}} \\
& \leq C^{j} t^{j}\left\|u_{0}\right\|_{L^{2}}\left(\left\|u_{0}\right\|_{\mathscr{F} L^{1}}^{2 j}+\left\|\phi_{n}\right\|_{\mathscr{F} L^{1}}^{2 j}\right) \\
& \lesssim C^{j} t^{j}\left\|u_{0}\right\|_{L^{2}}\left(R A^{d}\right)^{2 j} .
\end{aligned}
$$

Therefore, (3.12) follows from (3.14) and (3.15).

Next, we state a crucial proposition, establishing a lower bound on $\Xi_{1}\left(\phi_{n}\right)$. This proposition played an important role in establishing the norm inflation at the zero initial condition in [21] and will also play an important role in 
establishing norm inflation based at general initial data. The proof in [21] exploits the high-to-low energy transfer mechanism in $\Xi_{1}\left(\phi_{n}\right)$. We include the proof for readers' convenience.

Proposition 3.4. Let $\phi_{n}$ be as in (3.4) and $s<0$. Then, for $0<t \ll N^{-2}$, we have

$$
\left\|\Xi_{1}\left(\phi_{n}\right)(t)\right\|_{H^{s}} \gtrsim t R^{3} A^{2 d} \cdot f(A),
$$

where $f(A)$ is the function defined in (3.13).

First, recall the following simple lemma on the convolution of characteristic functions of cubes.

Lemma 3.5. Let $d \geq 1$. Then, there exists $c_{d}$ such that

$$
\mathbf{1}_{a+Q_{A}} * \mathbf{1}_{b+Q_{A}}(\xi) \geq c_{d} A^{d} \mathbf{1}_{a+b+Q_{A}}(\xi)
$$

for all $a, b, \xi \in \hat{\mathscr{M}}$ and $A \geq 1$. Here, $\hat{\mathscr{M}}$ denotes the Pontryagin dual of $\mathscr{M}$ defined in (1.4).

We now present the proof of Proposition 3.4.

Proof of Proposition 3.4. From (2.10) with (2.9), we have

$$
\Xi_{1}\left(\phi_{n}\right)(t)=i \int_{0}^{t} S\left(t-t^{\prime}\right)\left[S\left(t^{\prime}\right) \phi_{n} \overline{S\left(t^{\prime}\right) \phi_{n}} S\left(t^{\prime}\right) \phi_{n}\right] d t^{\prime} .
$$

Taking the Fourier transform, we have

$$
\begin{aligned}
& \mathscr{F}\left[\Xi_{1}\left(\phi_{n}\right)(t)\right](\xi)=i e^{i|\xi|^{2} t} \int_{\xi=\xi_{1}-\xi_{2}+\xi_{3}} \int_{0}^{t} e^{-i t^{\prime}\left(|\xi|^{2}-\left|\xi_{1}\right|^{2}+\left|\xi_{2}\right|^{2}-\left|\xi_{3}\right|^{2}\right)} d t^{\prime} \\
& \cdot \hat{\phi}_{n}\left(\xi_{1}\right) \overline{\hat{\phi}_{n}\left(\xi_{2}\right)} \hat{\phi}_{n}\left(\xi_{3}\right) d \xi_{1} d \xi_{2} \text {. }
\end{aligned}
$$

From (3.4), we have $\left|\xi_{j}\right| \lesssim N$ for $\xi_{j} \in \operatorname{supp} \hat{\phi}_{n}$. Then, with $\xi=\xi_{1}-\xi_{2}+\xi_{3}$, we have

$$
\left|t^{\prime}\left(|\xi|^{2}-\left|\xi_{1}\right|^{2}+\left|\xi_{2}\right|^{2}-\left|\xi_{3}\right|^{2}\right)\right| \ll 1
$$

for $0<t^{\prime} \ll N^{-2}$. Under the same condition, we have

$$
\operatorname{Re} \int_{0}^{t} e^{-i t^{\prime}\left(|\xi|^{2}-\left|\xi_{1}\right|^{2}+\left|\xi_{2}\right|^{2}-\left|\xi_{3}\right|^{2}\right)} d t^{\prime} \geq \frac{1}{2} t .
$$

Hence, it follows from (3.17), (3.19), and Lemma 3.5 with (3.4) that

$$
\left|\mathscr{F}\left[\Xi_{1}\left(\phi_{n}\right)(t)\right](\xi)\right| \gtrsim t R^{3} A^{2 d} \cdot \mathbf{1}_{Q_{A}}(\xi) .
$$

Lastly, noting that $\left\|\langle\xi\rangle^{s}\right\|_{L_{\xi}^{2}\left(Q_{A}\right)} \sim f(A)$, we obtain (3.16). 


\subsection{Proof of Proposition 3.1}

In this subsection, we prove Proposition 3.1. We claim that it suffices to show that, given $n \in N$, the following properties hold:

$$
\begin{aligned}
& \text { (i) } R A^{d / 2} N^{s} \ll \frac{1}{n}, \\
& \text { (ii) } T R^{2} A^{2 d} \ll 1, \\
& \text { (iii) } T R^{3} A^{2 d} \cdot f(A) \gg n, \\
& \text { (iv) } T R^{3} A^{2 d} \cdot f(A) \gg T^{2} R^{5} A^{4 d} \cdot f(A), \\
& \text { (v) } T \ll N^{-2}, \\
& \text { (vi) }(3.5) \text { and } R f(A) \gg\left\|u_{0}\right\|_{L^{2}}
\end{aligned}
$$

for some $A, R, T$, and $N$, depending on $n$. Here, $f(A)$ is as in (3.13). As before, implicit constants may depend on (fixed) $u_{0} \in \mathscr{S}(\mathscr{M})$.

We first show how these conditions (i)-(v) imply Proposition 3.1. The first condition (i) together with Lemma 3.2 verifies the first estimate in (3.1).

From (3.6) and (3.7) with (3.5), we have

$$
\left\|u_{0, n}\right\|_{\mathscr{F} L^{1}} \sim R A^{d} .
$$

Then, the second condition (ii) with Lemma 2.1 guarantees local existence of the solution $u_{n}$ on $[-T, T]$ with $\left.u_{n}\right|_{t=0}=u_{0, n}$ and Lemmas 2.3 and 2.5 yield the convergence of the power series expansion (3.8) in $C\left([-T, T] ; \mathscr{F} L^{1}\right)$.

Assuming the second conditions (ii) and (vi), Lemma 3.3 yields

$$
\begin{aligned}
\left\|\sum_{j=2}^{\infty} \Xi_{j}\left(u_{0, n}\right)(T)\right\|_{H^{s}} & \lesssim T^{2} R^{4} A^{4 d}\left\{R f(A)+\left\|u_{0}\right\|_{L^{2}}\right\} \\
& \sim T^{2} R^{5} A^{4 d} \cdot f(A) .
\end{aligned}
$$

Then, assuming the conditions (ii), (iii), (iv), (v), and (vi), it follows from Lemma 3.2, Proposition 3.4, and (3.21) with the power series (3.8) and (3.5) that

$$
\begin{aligned}
\left\|u_{n}(T)\right\|_{H^{s}} \geq & \left\|\Xi_{1}\left(\phi_{n}\right)(T)\right\|_{H^{s}}-\left\|\Xi_{0}\left(u_{0, n}\right)\right\|_{H^{s}} \\
& -\left\|\Xi_{1}\left(u_{0, n}\right)(T)-\Xi_{1}\left(\phi_{n}\right)(T)\right\|_{H^{s}}-\left\|\sum_{j=2}^{\infty} \Xi_{j}\left(u_{0, n}\right)(T)\right\|_{H^{s}}
\end{aligned}
$$




$$
\begin{aligned}
\gtrsim & T R^{3} A^{2 d} \cdot f(A)-\left(1+R A^{d / 2} N^{s}\right) \\
& -T R^{2} A^{2 d}\left\|u_{0}\right\|_{L^{2}}-T^{2} R^{5} A^{4 d} \cdot f(A) \\
\sim & T R^{3} A^{2 d} \cdot f(A) \gg n .
\end{aligned}
$$

This verifies the second estimate in (3.1) at time $t_{n}:=T$. Lastly, by choosing $N=N(n)$ sufficiently large, the condition (v) guarantees that $t_{n} \in(0,1 / n)$. This completes the proof of Proposition 3.1.

Therefore, it remains to verify the conditions (i)-(vi). We divide the argument into the following three cases: (1) $s<-d / 2$, (2) $s=-d / 2$, and (3) $-d / 2<s<0$. Note that the last case is relevant only for $d \geq 2$. Given $n \in N$, we first choose appropriate $A, R$, and $T$ in terms of $N$. Then, we choose $N=N(n) \gg 1$ so that all the conditions (i)-(vi) are satisfied. Note that the condition (iv) in fact follows from the condition (ii), and thus we only verify the condition (i), (ii), (iii), (v), and (vi) in the following.

Case 1: $s<-d / 2$. In this case, we set

$$
A=N^{(1 / d)(1-\delta)}, \quad R=N^{2 \delta}, \quad \text { and } \quad T=N^{-2-3 \delta},
$$

where $\delta>0$ is sufficiently small such that $s<-1 / 2-(3 / 2) \delta$. The conditions (v) and (vi) are trivially satisfied for $N \gg 1$. By choosing $N=N(n)$ sufficiently large, we have

$$
\begin{aligned}
R A^{d / 2} N^{s} & =N^{s+1 / 2+(3 / 2) \delta} \ll \frac{1}{n}, \\
T R^{2} A^{2 d} & =N^{-\delta} \ll 1, \\
T R^{3} A^{2 d} & =N^{\delta} \gg n,
\end{aligned}
$$

verifying the conditions (i), (ii), and (iii), respectively.

Case 2: $s=-d / 2$. In this case, we set

$$
A=\frac{N^{1 / d}}{(\log N)^{1 /(16 d)}}, \quad R=1, \quad \text { and } \quad T=\frac{1}{N^{2}(\log N)^{1 / 8}} .
$$

As before, the conditions (v) and (vi) are trivially satisfied for $N \gg 1$. By choosing $N=N(n)$ sufficiently large, we have

$$
\begin{aligned}
R A^{d / 2} N^{-d / 2} & =N^{(1 / 2)(1-d)}(\log N)^{-1 / 32} \ll \frac{1}{n}, \\
T R^{2} A^{2 d} & =(\log N)^{-1 / 4} \ll 1, \\
T R^{3} A^{2 d}(\log A)^{1 / 2} & \sim(\log N)^{-1 / 4}\left(\log N-\frac{1}{16} \log \log N\right)^{1 / 2} \sim(\log N)^{1 / 4} \gg n,
\end{aligned}
$$

verifying the conditions (i), (ii), and (iii), respectively. 
Case 3: $-d / 2<s<0$. Recall that this case is relevant only for $d \geq 2$. We set

$$
A=N^{2 / d-\delta}, \quad R=N^{-1-s+(d / 2) \delta-\theta}, \quad \text { and } \quad T=N^{-2+2 s+d \delta+\theta},
$$

where $\delta \gg \theta>0$ are sufficiently small such that

$$
-2 s>d \delta+\theta \quad \text { and } \quad-s \delta>2 \theta .
$$

It follows from (3.24) and (3.25) that the conditions (v) and (vi) are satisfied for $N \gg 1$. Moreover, by choosing $N=N(n)$ sufficiently large, we have

$$
\begin{aligned}
R A^{d / 2} N^{s} & =N^{-\theta} \ll \frac{1}{n}, \\
T R^{2} A^{2 d} & =N^{-\theta} \ll 1, \\
T R^{3} A^{2 d} \cdot A^{d / 2+s} & =N^{((-d+2) / d) s-2 \theta-s \delta} \geq N^{-2 \theta-s \delta} \gg n,
\end{aligned}
$$

verifying the conditions (i), (ii), and (iii), respectively.

Remark 3.6. In the following, we briefly discuss necessary modifications in proving Theorem 1.1 for the Wick ordered NLS (1.6) on $\boldsymbol{T}^{d}$. We first define the Duhamel integral operator $\tilde{\mathscr{I}}$ adapted to the renormalized nonlinearity in (1.6) by setting

$$
\tilde{\mathscr{I}}\left[u_{1}, u_{2}, u_{3}\right](t)=i \int_{0}^{t} S\left(t-t^{\prime}\right) \mathscr{N}\left[u_{1}\left(t^{\prime}\right), u_{2}\left(t^{\prime}\right), u_{3}\left(t^{\prime}\right)\right] d t^{\prime},
$$

where $\mathscr{N}$ is a trilinear operator given by

$$
\mathscr{F}\left(\mathscr{N}\left[f_{1}, f_{2}, f_{3}\right]\right)(\xi)=\sum_{\substack{\xi=\xi_{1}-\xi_{2}+\xi_{3} \\ \xi \neq \xi_{1}, \xi_{3}}} \hat{f}_{1}\left(\xi_{1}\right) \overline{\hat{f}_{2}\left(\xi_{2}\right)} \hat{f}_{3}\left(\xi_{3}\right)-\hat{f}_{1}(\xi) \overline{\hat{f}_{2}(\xi)} \hat{f}_{3}(\xi) .
$$

Then, one can go through Section 2 by replacing $\mathscr{I}$ with $\tilde{\mathscr{I}}$. In particular, Lemmas 2.5 and 2.6 hold with $\tilde{\mathscr{I}}$ in place of $\mathscr{I}$. This essentially follows from the following simple observation:

$$
\left|\mathscr{F}\left(\mathscr{N}\left[f_{1}, f_{2}, f_{3}\right]\right)(\xi)\right| \leq \sum_{\xi=\xi_{1}-\xi_{2}+\xi_{3}}\left|\hat{f}_{1}\left(\xi_{1}\right)\right|\left|\hat{f}_{2}\left(\xi_{2}\right)\right|\left|\hat{f}_{3}\left(\xi_{3}\right)\right| .
$$

As a consequence, Lemmas 3.2 and 3.3 also hold with $\tilde{\mathscr{I}}$ in place of $\mathscr{I}$. Lastly, let us consider Proposition 3.4 in this case. Let $\phi_{n}$ be as in (3.4), where $R=R(N)$ and $A=A(N)$ are as in (3.22), (3.23), or (3.24) with $N \gg 1$. In particular, we have $1 \ll A \ll N$. Then, it follows from (3.26) and (3.4) that

$$
\mathscr{F}\left(\mathscr{N}\left[\phi_{n}, \phi_{n}, \phi_{n}\right]\right)(\xi)=\sum_{\xi=\xi_{1}-\xi_{2}+\xi_{3}} \hat{\phi}_{n}\left(\xi_{1}\right) \overline{\hat{\phi}_{n}\left(\xi_{2}\right)} \hat{\phi}_{n}\left(\xi_{3}\right)=\mathscr{F}\left(\left|\phi_{n}\right|^{2} \phi_{n}\right)(\xi)
$$


for $\xi \in Q_{A}$. Hence, (3.20) holds. As a result, Proposition 3.4 also holds for $\tilde{\mathscr{I}}$. The discussion in Subsection 3.2 holds without any change.

Acknowledgments. T.O. was supported by the European Research Council (grant no. 637995 "ProbDynDispEq"). T.O. is grateful to Nobu Kishimoto for explaining his work in [21]. He would also like to thank Nikolay Tzvetkov for a conversation on Remark 1.6.

\section{References}

[1] Bejenaru, I. and Tao, T., Sharp well-posedness and ill-posedness results for a quadratic nonlinear Schrödinger equation, J. Funct. Anal., 233 (2006), 228-259.

[2] Bényi, Á., Oh, T. and Pocovnicu, O., Wiener randomization on unbounded domains and an application to almost sure well-posedness of NLS, Excursions in harmonic analysis, 4, 3-25, Appl. Numer. Harmon. Anal., Birkhäuser/Springer, Cham, 2015.

[3] Bényi, Á., Oh, T. and Pocovnicu, O., On the probabilistic Cauchy theory of the cubic nonlinear Schrödinger equation on $\boldsymbol{R}^{d}, d \geq 3$, Trans. Amer. Math. Soc. Ser. B, 2 (2015), $1-50$.

[4] Bourgain, J., Fourier transform restriction phenomena for certain lattice subsets and applications to nonlinear evolution equations I. Schrödinger equations, Geom. Funct. Anal., 3 (1993), 107-156.

[5] Bourgain, J., Invariant measures for the 2D-defocusing nonlinear Schrödinger equation, Comm. Math. Phys., 176 (1996), 421-445.

[6] Burq, N. and Tzvetkov, N., Random data Cauchy theory for supercritical wave equations. I. Local theory, Invent. Math., 173 (2008), 449-475.

[7] Burq, N. and Tzvetkov, N., Probabilistic well-posedness for the cubic wave equation, J. Eur. Math. Soc., 16 (2014), 1-30.

[ 8 ] Carles, R. and Kappeler, T., Norm-inflation for periodic NLS equations in negative Sobolev spaces, to appear in Bull. Soc. Math. France.

[9] Cazenave, T., Semilinear Schrödinger equations, Courant Lecture Notes in Mathematics, 10, New York University, Courant Institute of Mathematical Sciences, New York; American Mathematical Society, Providence, RI, 2003.

[10] Cazenave, T. and Weissler, F., Some remarks on the nonlinear Schrödinger equation in the critical case, Nonlinear semigroups, partial differential equations and attractors, 18-29, Lecture Notes in Math., 1394, Springer, Berlin, 1989.

[11] Choffrut, A. and Pocovnicu, O., Ill-posedness of the cubic half-wave equation and other fractional NLS on the real line, Internat. Math. Res. Not., (2016), https://doi.org/10.1093/ $\mathrm{imrn} / \mathrm{rnw} 246$.

[12] Christ, M., Power series solution of a nonlinear Schrödinger equation, Mathematical aspects of nonlinear dispersive equations, 131-155, Ann. of Math. Stud., 163, Princeton Univ. Press, Princeton, NJ, 2007.

[13] Christ, M., Colliander, J. and Tao, T., Asymptotics, frequency modulation, and low regularity ill-posedness for canonical defocusing equations, Amer. J. Math., 125 (2003), 1235-1293.

[14] Christ, M., Colliander, J. and Tao, T., Instability of the periodic nonlinear Schrödinger equation, arXiv:math/0311227v1 [math.AP]. 
[15] Christ, M., Colliander, J. and Tao, T., Ill-posedness for nonlinear Schrödinger and wave equations, arXiv:math/0311048 [math.AP].

[16] Christ, M., Colliander, J. and Tao, T., A priori bounds and weak solutions for the nonlinear Schrödinger equation in Sobolev spaces of negative order, J. Funct. Anal., 254 (2008), 368-395.

[17] Ginibre, J. and Velo, G., On the global Cauchy problem for some nonlinear Schrödinger equations, Ann. Inst. H. Poincaré Anal. Non Linéaire, 1 (1984), 309-323.

[18] Guo, Z. and Oh, T., Non-existence of solutions for the periodic cubic nonlinear Schrödinger equation below $L^{2}$, Internat. Math. Res. Not., (2016), doi: 10.1093/imrn/rnw271.

[19] Herr, S., Tataru, D. and Tzvetkov, N., Global well-posedness of the energy critical nonlinear Schrödinger equation with small initial data in $H^{1}\left(\boldsymbol{T}^{3}\right)$, Duke Math. J., 159 (2011) 329-349.

[20] Iwabuchi, T. and Ogawa, T., Ill-posedness for the nonlinear Schrödinger equation with quadratic non-linearity in low dimensions, Trans. Amer. Math. Soc., 367 (2015), 2613-2630.

[21] Kishimoto, N., A remark on norm inflation for nonlinear Schrödinger equations, preprint.

[22] Kenig, C., Ponce, G. and Vega, L., On the ill-posedness of some canonical dispersive equations, Duke Math. J., 106 (2001), 617-633.

[23] Koch, H. and Tataru, D., A priori bounds for the 1D cubic NLS in negative Sobolev spaces, Int. Math. Res. Not. IMRN 2007, no. 16, Art. ID rnm053, 36 pp.

[24] Koch, H. and Tataru, D., Energy and local energy bounds for the 1-d cubic NLS equation in $H^{-1 / 4}$, Ann. Inst. H. Poincaré Anal. Non Linéaire, 29 (2012), 955-988.

[25] Molinet, L., On ill-posedness for the one-dimensional periodic cubic Schrödinger equation, Math. Res. Lett., 16 (2009), 111-120.

[26] Oh, T. and Sulem, C., On the one-dimensional cubic nonlinear Schrödinger equation below $L^{2}$, Kyoto J. Math., 52 (2012), 99-115.

[27] Oh, T. and Wang, Y., On the ill-posedness of the cubic nonlinear Schrödinger equation on the circle, to appear in An. Ştiinţ. Univ. Al. I. Cuza Iaşi. Mat. (N.S.).

[28] Sulem, C. and Sulem, P.-L., The nonlinear Schrödinger equation. Self-focusing and wave collapse, Applied Mathematical Sciences, 139. Springer-Verlag, New York, 1999.

[29] Tao, T., Nonlinear dispersive equations. Local and global analysis, CBMS Regional Conference Series in Mathematics, 106. Published for the Conference Board of the Mathematical Sciences, Washington, DC; by the American Mathematical Society, Providence, RI, 2006.

[30] Tsutsumi, Y., $L^{2}$-solutions for nonlinear Schrödinger equations and nonlinear groups, Funkcial. Ekvac., 30 (1987), 115-125.

[31] Wang, Y., Periodic nonlinear Schrödinger equation in critical $H^{s}\left(\boldsymbol{T}^{n}\right)$ spaces, SIAM J. Math. Anal., 45 (2013), 1691-1703.

[32] Xia, B., Generic ill-posedness for wave equation of power type on 3D torus, arXiv:1507.07179 [math.AP].

\author{
nuna adreso: \\ School of Mathematics \\ The University of Edinburgh \\ James Clerk Maxwell Building \\ The King's Buildings, Peter Guthrie Tait Road \\ Edinburgh, EH9 3FD \\ United Kingdom \\ E-mail: hiro.oh@ed.ac.uk
}

(Ricevita la 16-an de septembro, 2015) 